\title{
The Origin of Biological Homochirality
}

\author{
Donna G. Blackmond \\ Department of Chemistry, The Scripps Research Institute, La Jolla, California 92037 \\ Correspondence: blackmond@scripps.edu
}

\section{SUMMARY}

The fact that sugars, amino acids, and the biological polymers they construct exist exclusively in one of two possible mirror-image forms has fascinated scientists and laymen alike for more than a century. Yet, it was only in the late 20th century that experimental studies began to probe how biological homochirality, a signature of life, arose from a prebiotic world that presumably contained equal amounts of both mirror-image forms of these molecules. This review discusses experimental studies aimed at understanding how chemical reactions, physical processes, or a combination of both may provide prebiotically relevant mechanisms for the enrichment of one form of a chiral molecule over the other to allow for the emergence of biological homochirality.

\section{Outline}

\section{Introduction}

2 Models for the origin of homochirality

3 Chemical models

4 Physical models
5 Combining physical and chemical approaches

6 Concluding remarks

References

Editors: Thomas R. Cech, Joan A. Steitz, and John F. Atkins

Additional Perspectives on RNA Worlds available at www.cshperspectives.org

Copyright (C 2019 Cold Spring Harbor Laboratory Press; all rights reserved; doi: 10.1101/cshperspect.a032540 


\section{INTRODUCTION}

The biopolymers that characterize life on Earth, and the molecular building blocks from which they are constructed, are both chiral and single-handed: that is, they have the potential to exist as either one of two nonsuperimposable mirror-image forms (Fig. 1), and they are selectively biosynthesized in only one of the two forms. Abiotic synthesis of chiral molecules in the absence of some type of chiral directing force produces an equal (racemic) mixture of both mirror-image molecules (enantiomers). The molecular "chirality" (a term originally coined by Kelvin 1904) of biological molecules is thus considered to be a fundamental signature of life (Blackmond 2010). Understanding how modern biology emerged from a presumably racemic prebiotic world represents an important step in understanding the origin of life.

In the history of chirality at both the molecular and macroscopic levels, investigations of chemical reactions that produce chiral molecules have been interwoven with studies of their physical properties. Although it is commonly agreed that the concept of molecular chirality was first introduced in Pasteur's famous 1848 physical separation of the mirror-image crystals of a tartrate salt (Pasteur 1848), the molecular basis of chirality was not in fact established for a further quarter century, when Le Bel and van't Hoff independently established the tetrahedral carbon atom (Le Bel 1874; van't Hoff 1874). This finding clarified that such an atom with four different substituents would be a chiral center with two mirror-image forms. Similarly, the physical phase behavior of amino acids was well-studied
(Roozeboom 1899) a half century before the macroscopic helicity of biopolymers was characterized (Pauling et al. 1951; Dunitz 2001), which in turn preceded our certain knowledge of which of the two hands of the monomers (called enantiomers) is favored by biology (Cahn et al. 1956).

RNA, DNA, and polypeptides all form supramolecular right-handed $\alpha$-helix structures, even while the amino acid molecules that make up peptides are designated as lefthanded (L), and the molecular building blocks that comprise RNA and DNA - the sugars ribose and deoxyribose-are called right-handed (D) (Fig. 1). Although the focus of RNA world chemistry tends to fall on these macroscopic structures without questioning how single-handedness arose at the molecular level, an important consideration is how the relative amounts of the two hands of the monomers, given as percent enantiomeric excess (ee) (\% ee $=100 \% *(\mathrm{D}-\mathrm{L}) /$ $(D+L))$, influences the production of the biopolymers. Polymerization of RNA is effectively inhibited if a monomeric nucleotide of the wrong chirality is ligated to a growing chain (Joyce et al. 1984). Proteins, in contrast, are rather more promiscuous; incorporation of amino acid residues of opposite chirality in nonribosomal peptides can occur and has even been shown in some cases to add structural and functional diversification as well as to impart chemical robustness. However, this opposite chirality is achieved not by incorporating unnatural D-amino acid molecular building blocks but through posttranslational conversion of the natural hand of an amino acid molecule in a peptide chain to its enantiomer (Kreil 1997). Thus, the establishment of a highly enantioenriched, if not enantiopure (ee $=100 \%$ ) pool of D-sugar and L-amino acid molecular building blocks would
A

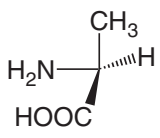

L-alanine

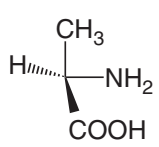

D-alanine

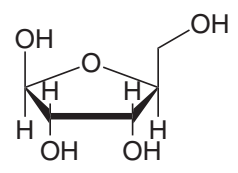

L-ribose

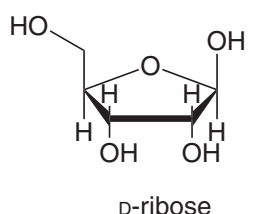

D-ribose

B
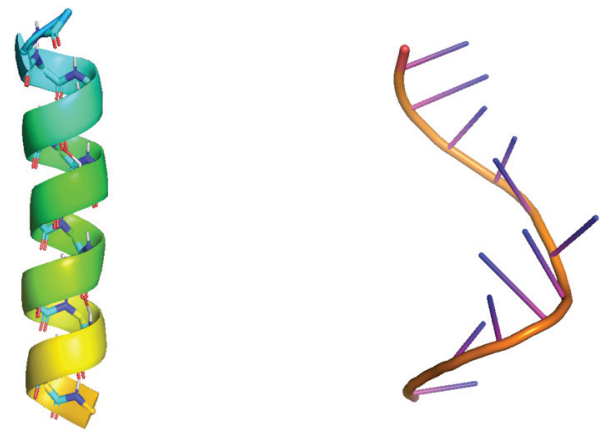

Figure 1. (A) Mirror-image molecules of alanine, a proteinogenic amino acid, and ribose, a pentose sugar. (B) Righthanded $\alpha$-helix structures of a peptide from amino acids (left) and RNA from ribonucleotides (right). 
appear to be a prerequisite for formation of the informational polymers implicated in the origin of life.

\section{MODELS FOR THE ORIGIN OF HOMOCHIRALITY}

Models for how homochirality might have emerged at the molecular level have been proposed since the early 1950s (Frank 1953). Interestingly, theoretical treatments preceded direct experimental investigations by nearly half a century. The question has two parts. The first concerns "symmetry breaking": What could have served as the original template for biasing production of one enantiomer over the other in the prebiotic world? The second concerns "amplification": How could a small imbalance of enantiomers be sustained and propagated to give us the biological world of single chirality that surrounds us?

\subsection{Symmetry Breaking}

Symmetry breaking is a term that has different meanings in different branches of science. To a physicist, it is a phenomenon in which infinitesimally small fluctuations acting on a system decide the system's fate by choosing which branch of a bifurcation is taken when crossing a critical point. To an outside observer unaware of the fluctuations (or "noise"), the choice will appear arbitrary. To a biologist, symmetry breaking is the process by which uniformity or invariance in a system is broken, linked to functional diversification from molecular assemblies to subcellular structures to cell types. To a chemist, symmetry breaking is the spontaneous generation of an excess of one enantiomer of a chiral molecule over the other in the absence of an external chiral source.

The chemist's definition of chirality meets that of the physicist's in the concept of a parity inversion, an operation that transforms a physical phenomenon (or a molecule) into its mirror image. Parity is conserved in electromagnetism, gravity, and strong interactions, but in the mid-20th century, it was discovered that parity is not conserved ("parity violation") in weak interactions. One of the consequenc- es of parity violation is that a very small energy difference exists between enantiomers that previously had been considered identical. This finding led many to consider possible implications for the origin of biological homochirality. Work aimed at estimating the magnitude of this (exceedingly small) energy difference is ongoing, and although the question is not yet settled (Quack 2002), a relationship between biological homochirality and parity violation energy difference of enantiomers is not yet supported by either theoretical or experimental findings.

\subsection{Chiral Amplification}

Evidence of small enantiomeric excesses toward L-amino acids found in chondritic meteorite deposits (Elsila et al. 2016) allows the hypothesis that the initial imbalance between enantiomers on earth may have been seeded from an extraterrestrial source. A small imbalance is, however, a necessary but not sufficient condition to explain the emergence of homochirality, which requires some type of amplification or propagation mechanism following symmetry breaking. Thus, whether symmetry breaking to provide an imbalance in enantiomers was preordained or came about by chance, arising on earth or elsewhere, an amplification mechanism remains the key to increasing enantiomeric excess and ultimately to approaching the homochiral state. Experimental studies aimed at uncovering such processes, which began in earnest in the last decade of the 20th century, may be divided into either chemical or physical processes. The remainder of this review will highlight some of these experimental findings.

\section{CHEMICAL MODELS}

Studies exploring how enantioenrichment may result from chemical transformations fall into a number of categories, including autocatalytic "far-from-equilibrium" chiral amplification (Fig. 2) and reactions mediated by chiral catalysts or chiral auxiliaries (Fig. 3). The latter group of reactions includes the formose carbohydrate synthesis, the Strecker

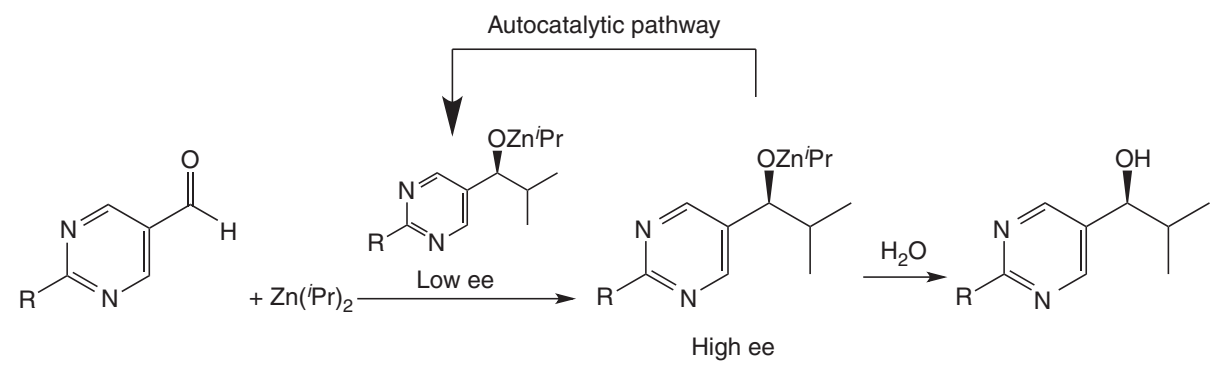

Figure 2. Autocatalytic amplification of enantiomeric excess in the Soai alkylation of pyrimidyl aldehydes. 
D.G. Blackmond

\section{A Formose reaction:}

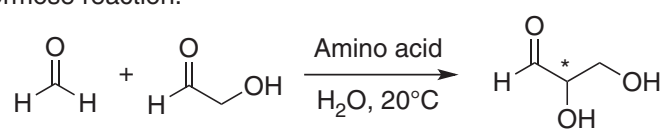

B Strecker reaction:

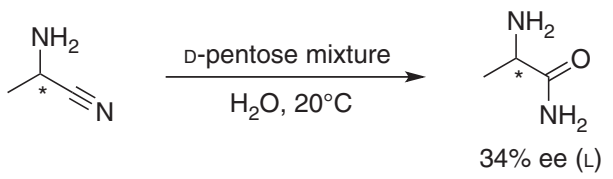

C Powner/Sutherland RNA synthesis:

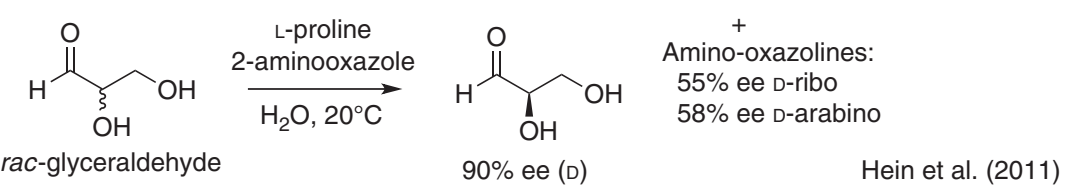

D Breslow chirality transfer via transamination:

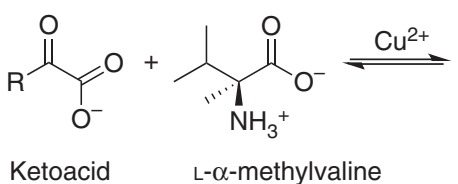

Ketoacid<smiles>[R]C([NH3+])C(=O)[O-]</smiles>

$\alpha$-amino acid $37 \%$ ee $(\llcorner)$

Figure 3. Prebiotically plausible reactions showing enantioenrichment of either sugars or amino acids.

amino acid synthesis, the Powner/Sutherland synthesis of RNA precursors, and chirality transfer reactions such as transaminations, and all involve chemistry that might occur under prebiotically plausible conditions. In contrast, the Soai alkylation of pyrimidyl aldehydes (Soai et al. 1995), which is the sole documented example of chiral amplification via autocatalysis, requires conditions far removed from those thought to exemplify the prebiotic world. However, because this reaction provided the first experimental proofof-concept of earlier theoretical proposals, its study serves as a model for understanding how homochirality might have emerged and may help us search for prebiotically plausible reactions with similar characteristics.

\subsection{Homochirality via Asymmetric Autocatalysis}

In 1953 (a good year for research reports related to the origin of life), a mathematical model for the evolution of homochirality was proposed based on a chiral molecule that catalyzes its own self-production and at the same time acts to suppress synthesis of its enantiomer (Frank 1953). The last sentence of this theoretical work, which captivated several generations of chemists, contained an exhortation to experimental chemists: "a laboratory demonstration may not be impossible." More than 40 years passed before Soai and coworkers met this challenge with a report of the autocatalytic alkylation of pyrimidyl aldehydes with diisopropylzinc (Soai et al. 1995). The rate of the reaction is not only accelerated by addition of catalytic amounts of its own product, but this autocatalytic product may ultimately be formed in very "high" enantiomeric excess starting from a very "low" enantiomeric excess in the original catalyst (Fig. 2). Since this initial discovery, Soai's group has gone on to present remarkable further observations of asymmetric amplification in the reaction that now bears his name. Enantiomeric excesses as high as $85 \%$ were reported for a reaction initiated with a $0.1 \%$ ee mixture of chiral molecules produced from exposure to circularly polarized light (Shibata et al. 1998). Asymmetric amplification has also been observed for the reaction initiated by inorganic chiral materials such as quartz (Soai et al. 1999). Most recently, Soai has shown that the reaction may be selectively triggered solely by the minute mirror-image difference provided by isotope chirality of an initiator molecule (Kawasaki et al. 2009), showing that the reaction needs only an extremely small nudge to direct it consistently to the left or to the right.

The first mechanistic rationalization of this extraordinary reaction was reported in 2001 (Blackmond et al. 2001). 

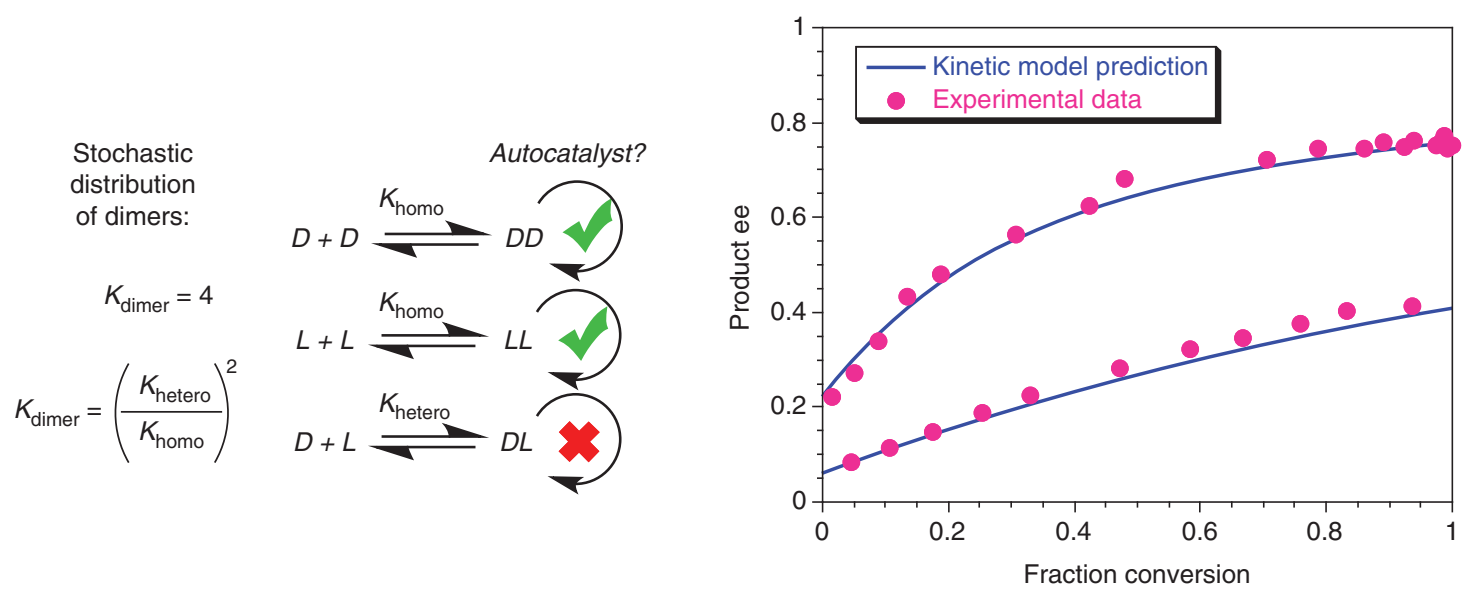

Figure 4. Blackmond/Brown model (left) and product enantiomeric excess as a function of fraction conversion of substrate (right) predicted from reaction rate profiles fit to the kinetic model (blue lines) and corroborated by analysis of experimental enantiomeric excess (ee) data.

A kinetic model developed based on highly accurate in situ measurements of the reaction's progress independently predicted both the temporal degree of asymmetric amplification, confirmed by compositional analysis, and the relative concentrations of the catalyst species, confirmed by nuclear magnetic resonance (NMR) spectroscopy. The reaction product dimerizes stochastically to form the active autocatalyst in the case of homochiral dimers (DD and LL), with the heterochiral dimer (DL) as an inactive reservoir. Figure 4 shows how the kinetic model compares with experimental data for asymmetric amplification in the Soai reaction performed out with two different initial catalyst ee values. This work showed that the Soai reaction couples autocatalysis with a form of "mutual antagonism" between enantiomers, thus evoking the main features of the Frank model. Further mechanistic studies have refined this reaction as a model for symmetry breaking and autocatalytic amplification of ee. The structure and possible role of tetrameric catalysts first proposed by Brown and coworkers (Gridnev and Brown 2004) was supported by kinetic and spectroscopic measurements by Blackmond, Brown, and coworkers (Buono and Blackmond 2003, Quaranta et al. 2010) before Soai and coworkers (Matsumoto et al. 2015) ultimately obtained a crystal structure that confirmed the tetrameric species first proposed by Brown. Most recently, a mechanistic rationalization for the role of isotopically chiral molecules in the Soai reaction has shed light on both the processes of symmetry breaking and asymmetric amplification via autocatalysis (Hawbaker and Blackmond 2018).

Because small-molecule autocatalysis may be thought of as the simplest form of self-replication, it remains an appealing scenario for the RNA community accustomed to believing about macromolecular self-replication. The discovery of a prebiotically relevant reaction, or a network of reactions, that obey the precepts of Frank model autocatalysis remains something of a "Holy Grail" for researchers probing the origin of biological homochirality (Blackmond 2004).

\subsection{Reactions Mediated by Chiral Entities}

A number of different transformations have been documented that produce enantioenriched sugar or amino acid building blocks under conditions with greater prebiotic relevance than the Soai autocatalytic system discussed above. Two venerable reactions known for more than a century and a half provide routes to the two classes of biological molecules for which chirality is key: the formose sugar synthesis, discovered in 1861 (Butlerow 1861) and unveiled mechanistically by Breslow in 1959 (Breslow 1959), and the Strecker amino acid synthesis discovered in 1850 (Strecker 1850). Although most reports do not address chirality, recent studies have begun to show how chiral catalysts or auxiliaries could provide partial product enantioenrichment. Interestingly, these reactions invoke a kind of synergy between the sugars and amino acids, as one of these two classes of molecule helps to direct enantioselectivity in the synthesis of the other: amino acids act as catalysts to enantioenrich sugars in the formose reaction (Fig. 3A), and sugars enantioselectively resolve amino acid precursors in the Strecker hydration of aminonitriles (Fig. 3B).

The Strecker reaction between aldehydes, cyanide, and ammonia represents a prebiotically plausible route to amino acids, forming first a racemic mixture of aminonitriles. Directing the hydration of these molecules in a dynamic kinetic resolution has been proposed as a pathway for enan- 
tioenrichment, and C4-C6 aldehydic carbohydrates can act as catalysts in this transformation. Derivatives of these sugars have been found in meteorites with significant excesses toward the natural D-sugars. Strikingly, mixtures of the natural D-pentoses produce enantioenriched L-aminoamides, which are then further hydrolyzed to the natural L-amino acids (Fig. 3B). This work reiterates the "systems chemistry" aspect of emergence of enantioenrichment from complex mixtures, and it hints at a prebiotic role for other pentose sugars that would have been present in quantities similar to ribose and deoxyribose before these sugars were ultimately chosen for their role in biological polymers (Wagner et al. 2016).

The Powner/Sutherland synthesis of RNA (Powner et al. 2009) represented a leap forward for the RNA world hypothesis because it showed for the first time a synthesis of activated RNA nucleotide monomers from simple prebiotically plausible feedstock molecules. Starting from D-glyceraldehyde, the synthesis bypasses the problematic step of combining ribose with a nucleobase. The work also highlighted "systems chemistry" aspects of emergent reactivity in complex mixtures. Enantioenrichment in this reaction network was established later when it was shown (Hein et al. 2011) that natural L-amino acids preferentially sequester unnatural L-glyceraldehyde, leaving D-glyceraldehyde free for RNA synthesis (Fig. 3C). Even partial enantioenrichment suffices to produce homochiral RNA building blocks; enantiopure crystals of the ribo-amino-oxazoline naturally precipitate from reaction mixtures above $20 \%$ ee.

Transfer of chirality from nonracemic meteoritic amino acids to proteinogenic amino acids was shown by Breslow and coworkers (Levine et al. 2008) in transamination reactions catalyzed by $\mathrm{Cu}$ ions (Fig. 3D). This work suggests how chirality first seeded extraterrestrially could provide some level of enantioenrichment in the building blocks of life. However, because the enantiomeric excess of the reaction products is lower than that of the molecules from which they are transferred, further amplification mechanisms would be required to produce significant enantioenrichment.

\section{PHYSICAL MODELS}

Understanding how the physical properties of chiral molecules might be exploited for enantioenrichment requires an understanding of their physical phase behavior-their solubilities and the manner in which they crystallize (Jacques et al. 1994). These concepts, which have been studied extensively for more than a century, are illustrated in Figure 5. When both enantiomers of a molecule are present in solution, crystallization proceeds either with both enantiomers mixed in a single crystal or with enantiomers forming separate crystals. The formation of separate D and L crystals, as in those Pasteur separated with his tweezers, are known as "conglomerates" (Fig. 5, right panel). When the mixed enantiomers form a regular 1:1 D:L matrix, the molecule is called a "racemic compound" (Fig. 5, left panel). The difference in the two types of crystals comes about as a result of characteristics of the molecules that make their solid phase stability greater with heterochiral interactions or homochiral interactions. The former makes up 90\% of all known chiral compounds, and indeed 17 of the 19 proteinogenic amino acids are racemic compounds.

When a system containing an unequal number of $\mathrm{D}$ and $\mathrm{L}$ molecules in a solvent reaches equilibrium between solid and solution phase molecules, the Gibbs phase rule for a ternary system (two enantiomers and one solvent) with three phases (two solid phases and one solution phase) dictates that, at fixed temperature and pressure, the solution composition is fixed at a composition characteristic of that system known as its eutectic composition. An important implication of the phase rule is that a partitioning of enantiomers occurs between the solution and solid in both cases. For conglomerates, because the solubilities of the two enantiomers are identical, the saturated solution will contain an equal number of $\mathrm{D}$ and $\mathrm{L}$ molecules regardless of how great the overall imbalance between $D$ and $L$, suggesting the potential for enantioenrichment in the solid phase. For racemic compounds with an unequal number of $\mathrm{D}$ and $\mathrm{L}$ molecules, the solid phase will form the heterochiral

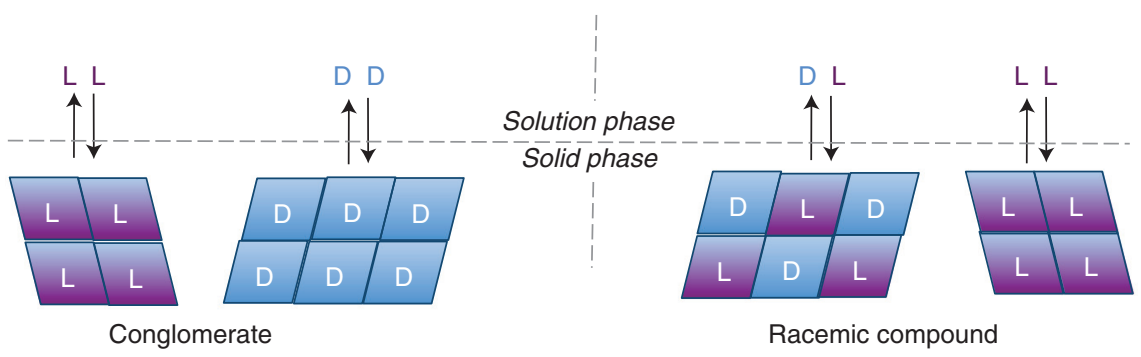

Figure 5. Equilibrium between chiral crystals and their saturated solution phases for systems comprised of an unequal number of $\mathrm{D}$ and L molecules. (Right) Racemic compound, in which heterochiral interactions are stronger than homochiral interactions; (left) conglomerate, in which homochiral interactions dominate. 
1:1 D:L solid with all of the minor enantiomer present in the solid phase, while the remaining solid phase molecules of the major enantiomer will be left to form homochiral crystals. The relative solubility of the D:L solid compared with that of the homochiral solid dictates the magnitude of the solution phase imbalance between $\mathrm{D}$ and $\mathrm{L}$. In this case, enantioenrichment in the solution phase is common. Below, we discuss models developed for the emergence of homochirality for each type of crystal in turn.

\section{1 "Eve Crystal" Model for Conglomerates}

It has been known for more than a hundred years that certain molecules such as $\mathrm{NaClO}_{3}$ are achiral in solution but crystallize as conglomerates, typically forming equal amounts of left and right-handed crystals (Kipping and Pope 1898). Under some conditions, the system breaks symmetry during the crystallization and produces more of one hand than the other, even in the absence of a detectable chiral source. The most striking example of this phenomenon was reported by Kondepudi (Kondepudi et al. 1990) who showed that when a homogeneous solution of achiral $\mathrm{NaClO}_{3}$ was cooled to initiate crystallization, the process could be directed if it was accompanied by rapid stirring. In this case, all of the crystals formed were of single chirality, randomly left-handed or right-handed in repeated experiments. This was rationalized by considering the dynamics of crystallization: Formation of the very first crystal (primary nucleation) from a homogeneous supersaturated solution is energetically unfavorable, although crystal growth by adding molecules to crystals already present (secondary nucleation) occurs much more readily. Under rapid stirring, if the first crystal formed, or "Eve" crystal, is broken by into thousands of smaller crystals by impact with the stirring bar, all of these "daughter" crystals are necessarily of the same chiral form, and they grow rapidly by secondary nucleation from solution molecules. If secondary nucleation occurs fast enough before nucleation of any new primary crystals, a single chiral solid state may result (McBride and Carter 1991). This phenomenon, shown in Figure 6 bears resemblance to "far-from-equilibrium" autocatalytic reaction processes such as the Soai reaction discussed earlier.

\subsection{Attrition-Enhanced Deracemization of Conglomerates}

The same achiral salt $\mathrm{NaClO}_{3}$ became the subject of another intriguing process resulting in solid phase homochirality about fifteen years after Kondepudi's Eve crystal model was introduced. A report by Viedma (Viedma 2005) described a remarkable experiment that starts at what would be the end of a conventional crystallization with an equal number of right- and left-hand crystals of $\mathrm{NaClO}_{3}$ in saturated aqueous solution. Because the system is not supersaturated, no new crystals nucleate; the only processes occurring in the flask are the continual dissolution and reaccretion of $\mathrm{NaClO}_{3}$ molecules to and from existing crystals, and these rates are balanced at equilibrium. Thermodynamics tells us that no net change should occur, and that is exactly what has been found in such experiments for more than a hundred years. Viedma changed all this when he observed that the system evolved over time to "one" of the two chiral solids in a random fashion, provided that he added glass beads to the stirred vial. Attrition by glass beads produces a greater number of smaller crystals, which show higher solubility according to the Gibbs-Thomson rule, causing a slight supersaturation of $\mathrm{NaClO}_{3}$ in solution. Not sufficiently supersaturated to support primary nucle-

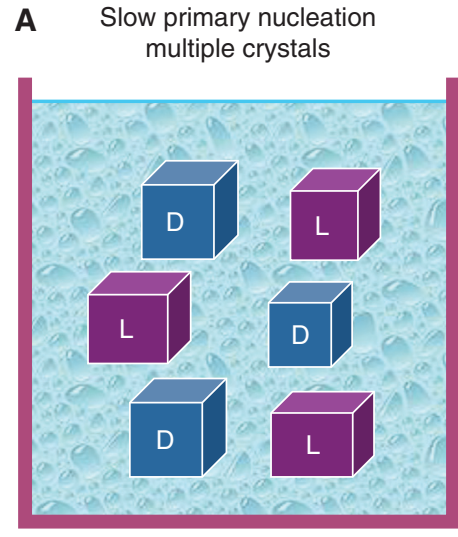

No stirring
B Rapid secondary nucleation from shear of primary crystal

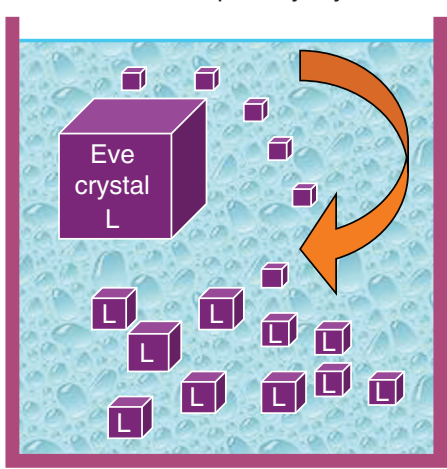

Rapid stirring

Figure 6. Effect of stirring on rates of primary $(A)$ versus secondary $(B)$ nucleation in the "Eve crystal" model of conglomerate-forming $\mathrm{NaClO}_{3}$. 


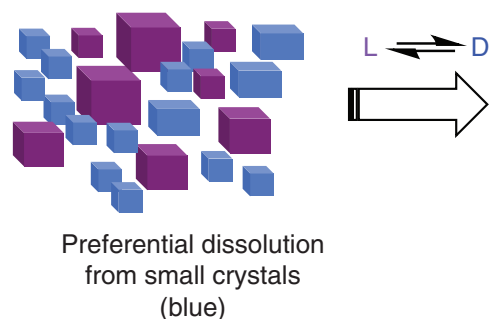
(blue)

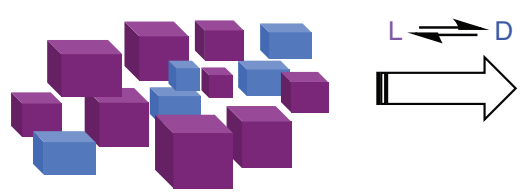

Preferential reaccretion onto large crystals (purple)

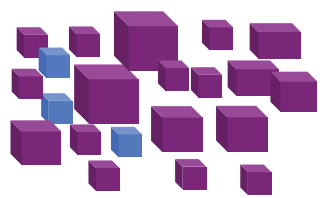

Eventual disappearance of small crystals (blue)

Figure 7. Evolution of solid phase homochirality based on dissolution and reaccretion of molecules from small crystals (shown in blue) of one enantiomer onto larger crystals (shown in purple) of the other enantiomer, through the conduit of solution phase interconversion between enantiomers.

ation, the system strives to redress the balance between solid and solution by increasing the rate of reaccretion of solution phase $\mathrm{NaClO}_{3}$ onto existing crystals. A key point is that once a molecule of $\mathrm{NaClO}_{3}$ dissolves from a crystal, it no longer possesses chirality and it retains no memory of the chiral form it previously showed as part of a crystal. Solution-phase $\mathrm{NaClO}_{3}$ thus has no preference for reaccreting to a left- or right-handed crystal. What solution phase molecules do have, however, is a preference for adding to larger crystals over smaller ones, a phenomenon known as Ostwald ripening. If, by chance, the system contains a predominance of large crystals of one hand, solution phase $\mathrm{NaClO}_{3}$ will preferentially add to these crystals, and the quantity of this enantiomorphic solid will increase relative to its mirror-image form (Fig. 7). Evolution of solid phase homochirality in this case requires only a chance imbalance in the crystal size of left versus right-hand crystals, with the solution phase serving as the conduit through which the molecules forget their solid-state chiral history and are free to choose a new solid-state chiral destiny.

These results led many to consider a possible extension of this process from the achiral $\mathrm{NaClO}_{3}$ to systems more relevant to the origin of life, intrinsically chiral molecules that form conglomerate solids. In this case, the solution conduit between the two solids arises by the process through which chiral molecules rapidly interconvert between their left and right forms, known as racemization. This was successfully shown first for an amino acid derivative (Noorduin et al. 2008) and then for the proteinogenic amino acid aspartic acid (Viedma et al. 2008).

\subsection{Crystal Engineering of Racemic Compounds}

Morowitz was the first to recognize that solution-phase enantioenrichment is possible for racemic compounds if the heterochiral crystal is relatively insoluble compared with the homochiral crystal (Morowitz, 1969). This work was elaborated by measuring the eutectic composition for a variety of amino acids and nucleosides (Klussmann et al. 2006a; Breslow and Levine 2006; Breslow and Cheng 2009). The degree of chiral amplification is dictated by the thermodynamic properties of the particular compound. The proteinogenic amino acid serine shows a eutectic of 99\% ee, meaning that a nearly homochiral solution is achievable even when the overall ee of the solid and solution combined is close to racemic. Valine, on the other hand, shows a eutectic of $47 \%$ ee, which means that a much lower level of chiral amplification is possible for this amino acid. Further work discovered that the cocrystallization of a variety of small molecules with amino acids can alter the relative heterochiral/homochiral solubility and thus "engineer" the eutectic composition (Klussmann et al. 2006b; 2007). This finding helped to generalize the eutectic model, revealing a number of prebiotically plausible cocrystallizations that raised the eutectic composition of amino acids. For example, when valine cocrystallizes with fumaric acid, its eutectic increases to $99 \%$ ee, affording a path to solution-phase homochirality. Similarly, proline's eutectic composition increases from $50 \%$ ee to $99 \%$ ee when chloroform cocrystallizes with the racemic compound (Fig. 8). Similar concepts have been invoked to rationalize enantioenrichment during sublimation of amino acids (Fletcher et al. 2007).

\section{COMBINING PHYSICAL AND CHEMICAL APPROACHES}

A number of examples illustrate how prebiotic chemistry resulting in amplification of enantiomeric excesses of amino acids and sugars has been aided by physical processes. The selective thermodynamic partitioning of amino acid enantiomers between solution and solid phases has been invoked as a possible prebiotic mechanism for solution phase amplification of enantiomeric excess.

A combination of these physical and chemical amplification processes was shown for the proline-mediated reaction of glyceraldehyde and concomitant production of enantioenriched ribo-amino-oxazoline (Fig. 4C). Enantioenrichment of $1 \%$ ee L-proline was achieved by forming 

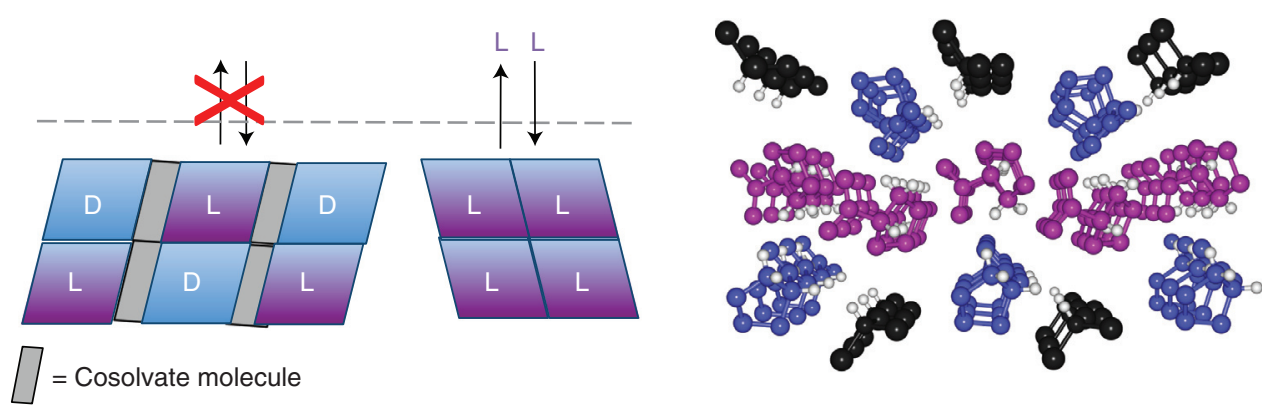

Figure 8. Cosolvate enriches the solution phase enantiomeric excess by suppressing the solutility of the racemic compound. (Right) Crystal structure of LD proline with $\mathrm{CH}_{3} \mathrm{Cl}$.

the $\mathrm{CHCl}_{3}$-DL-proline cosolvate (Klussmann et al. 2006b). Nearly enantiopure solution phase proline was recovered and mixed with racemic glyceraldehyde and 2-amino-oxazole in aqueous solution to produce enantioenriched riboand arabino-amino-oxazolines in solution, from which enantiopure crystals of the ribo-amino-oxazoline precipitated (Hein et al. 2011). Thus, chemical amplification of ee is augmented by two physical amplification processes: enantioenrichment of the amino acid before the chemical reaction, and the selective crystallization of enantiopure ribo-amino-oxazoline following the chemical reaction.

A likely prebiotic scenario suggests that a complex pool of organic molecules would likely be present in any potential reaction medium. To test the robustness of these chirality amplification processes in multicomponent mixtures, the reaction of racemic glyceraldehyde with 2-amino-oxazole was performed in mixtures of a variety of different amino acids. Enantiopure crystals of ribo-amino-oxazoline were successfully obtained directly from crude reaction mixtures containing as many as fourteen of the nineteen chiral, proteinogenic amino acids (Hein et al. 2011). The emergence of molecules of single chirality from complex, multicomponent mixtures is a proof of "systems chemistry" (von Kiedrowski 2005) and supports the robustness of the processes under potential prebiotic conditions.

\section{CONCLUDING REMARKS}

The implications of the single chirality of biological molecules may be viewed in the context of increasing complexity at both the molecular and macroscopic levels. In the molecular sciences research, this has been codified as the field of "systems chemistry" (von Kiedrowski, 2005), which seeks to understand the chemical roots of biological organization by studying the emergence of system properties that may be different from those showed individually by the components in isolation. The abiotic construction of homochiral genetic polymers from molecular building blocks implies at least some minimal degree of enantioenrichment at the molecular level, if not molecular homochirality, to ensure efficiency as RNA or peptide chains grow in length. This review has described a variety of work invoking either chemical or physical processes, or both, that might allow the emergence of biological homochirality.

\section{REFERENCES}

Blackmond DG. 2004. Asymmetric autocatalysis and its implications for the origin of homochirality. Proc Natl Acad Sci 101: 5732-5736.

Blackmond DG. 2010. The origin of biological homochirality. Cold Spring Harb Perspect Biol 2: a002147.

Blackmond DG, McMillan CR, Ramdeehul S, Schorm A, Brown JM. 2001. Origins of asymmetric amplification in autocatalytic alkylzinc additions. J Am Chem Soc 123: 10103-10104.

Breslow R. 1959. On the mechanism of the formose reaction. Tetrahedron Lett 22-36.

Breslow R, Cheng Z-L. 2009. On the origin of terrestrial homochirality for nucleosides and amino acids. Proc Natl Acad Sci 106: 9144-9146.

Breslow R, Cheng Z-L. 2010. L-Amino acids catalyze the formation of an excess of D-glyceraldehyde, and thus of other D sugars, under credible prebiotic conditions. Proc Natl Acad Sci 107: 5723-5725.

Breslow R, Levine M. 2006. Amplification of enantiomeric concentrations under credible prebiotic conditions. Proc Natl Acad Sci 103: 1297912980.

Buono FG, Blackmond DG. 2003. Kinetic evidence for a tetrameric transition state in the asymmetric alkylation of pyrimidyl aldehydes. J Am Chem Soc 125: 8978-8979.

Butlerow A. 1861. Bildung einer zuckerartigen substanz durch synthese. Justus Liebigs Annalen der Chemie 120: 295-298.

Cahn RS, Ingold CK, Prelog V. 1956. The specification of asymmetric configuration in organic chemistry. Experientia 12: 81-94.

Dunitz JD. 2001. Pauling's left-handed $\alpha$-helix. Angew Chemie Int Ed. 40: 4167-4173.

Elsila JE, Aponte JC, Blackmond DG, Burton AS, Dworkin JP, Glavin DP. 2016. Meteoritic amino acids: Diversity in compositions reflects parent body histories. ACS Central Sci 2: 370-379.

Fletcher SP, Jagt RBC, Feringa BL. 2007. An astrophysically-relevant mechanism for amino acid enantiomer enrichment. Chem Commun $2578-2580$.

Frank FC. 1953. On spontaneous asymmetric synthesis. Biochimica et Biophysica Acta 11: 459-463.

Gridnev ID, Brown JM. 2004. Solution structure and reagent binding of the zinc alkoxide catalyst in the Soai asymmetric autocatalytic reaction. Proc Natl Acad Sci 101: 5727-5731.

Hawbaker N, Blackmond DG. 2018. Rationalization of asymmetric amplification via autocatalysis triggered by isotopically chiral molecules. ACS Cent Sci doi: 10.1021/acscentsci.8b00297. 
Hein JE, Blackmond DG. 2012. On the origin of single chirality of amino acids and sugars in biogenesis. Acc Chem Res 45: 2045-2054.

Hein JE, Tse E, Blackmond DG. 2011. A route to enantiopure RNA precursors from nearly racemic starting materials. Nat Chem 3: 704706.

Jacques J, Collet A, Wilen SH. 1994. Enantiomers, racemates and resolution. Krieger, Malabar, FL.

Joyce GF, Visser GM, van Boekel CAA, van Boom JH, Orgel LE. 1984. Chiral selection in poly $(\mathrm{C})$-directed synthesis of oligo(G). Nature 310: 602-604.

Kawasaki T, Matsumura Y, Tsutsumi T, Suzuki K, Ito M, Soai K. 2009. Asymmetric autocatalysis triggered by carbon isotope (13C/12C) chirality. Science 324: 492-495.

Kelvin WT. 1904. Baltimore lectures on molecular dynamics and the wave theory of light. C.J. Clay, London.

Kipping WS, Pope WJ. 1898. Enantiomorphism. J Chem Soc Trans 73: 606-617.

Klussmann M, Iwamura H, Mathew SP, Wells DH Jr, Pandya U, Armstrong A, Blackmond DG. 2006a. Thermodynamic control of asymmetric amplification in amino acid catalysis. Nature 441: 621-623.

Klussmann M, White AJP, Armstrong A, Blackmond DG. 2006b. Rationalization and prediction of solution enantiomeric excess in ternary phase systems. Angew Chem Int Ed 47: 7985-7989.

Klussmann M, Izumi T, White AJP, Armstrong A, Blackmond DG. 2007. Emergence of solution-phase homochirality via crystal engineering of amino acids. J Am Chem Soc 123: 7657-7660.

Kondepudi DK, Kaufman RJ, Singh N. 1990. Chiral symmetry breaking in sodium chlorate crystallization. Science 250: 975-976.

Kreil G. 1997. D-amino acids in animal peptides. Annu Rev Biochem 66: 337-345.

Le Bel JA. 1874. Sur des relations qui existent entre les formules atomiques des corps organiques et la pouvoir rotatoire de leur dissolutions. Bull Soc Chim Fr 22: 337-347.

Levine M, Kenesky CS, Mazori D, Breslow R. 2008. Enantioselective synthesis and enantiomeric amplification of amino acids under prebiotic conditions. Org Lett 10: 2433-2436.

Matsumoto A, Abe T, Hara A, Tobita T, Sasagawa T, Kawasaki T, Soai K. 2015. Crystal structure of the isopropylzinc alkoxide of pyrimidyl alkanol: Mechanistic insights for asymmetric autocatalysis with amplification of enantiomeric excess. Angew Chemie Int Ed 127: 1543315436.

McBride JM, Carter RL. 1991. Spontaneous resolution by stirred crystallization. Angew Chem Intl Ed 30: 293-295.

Morowitz M. 1969. A mechanism for the amplification of fluctuations in racemic Mixtures. J Theor Biol 25: 491-494.

Noorduin WL, Izumi T, Millemaggi A, Leeman M, Meekes H, Van Enckevort WJP, Kellogg RM, Kaptein B, Vlieg E, Blackmond DG. 2008.
Emergence of a single solid chiral state from a nearly racemic amino acid derivative. J Am Chem Soc 130: 1158-1159.

Pasteur L. 1848. Relation qui peut exister entre la forme crystalline et la compostion chimique, et sur las cause de la polarization rotatoire. Ann Chim Phys 26: 535-538.

Pauling L, Corey RB, Branson HR. 1951. The structure of proteins: Two hydrogen-bonded helical configurations of the polypeptide chain. Proc Natl Acad Sci 37: 205-211.

Powner MW, Gerland B, Sutherland JD. 2009. Synthesis of activated pyrimidine ribonucleotides in prebiotically plausible conditions. $\mathrm{Na}$ ture 459: 239-242.

Quack M. 2002. How important is parity violation for molecular and biomolecular chirality? Angew Chem Int Ed 41: 4618-4630.

Quaranta M, Gehring T, Odell B, Brown JM, Blackmond DG. 2010. Unusual inverse temperature dependence on reaction rate in the asymmetric autocatalytic alkylation of pyrimidyl aldehydes. J Am Chem Soc 132: 15104-15107.

Roozeboom HWB. 1899. Löslichkeit und schmelzpunkt als kriterion für racemische verbindungen, speuoracemische mischkrystalle und inactive conglomerate. $Z$ Phys Chem 28: 494-517.

Shibata J, Yamamoto T, Matsumoto N, Yonekubo S, Osanai S, Soai K. 1998. Amplification of a slight enantiomeric imbalance in molecules based on asymmetric autocatalysis. The first correlation between high enantiomeric enrichment in a chiral molecule and chircularly polarized light. J Am Chem Soc 120: 12157-12158.

Soai K, Shibata T, Morioka H, Choji K. 1995. Asymmetric autocatalysis and amplification of enatiomeric excess of a chiral molecule. Nature 378: 767-768.

Soai K, Osanai S, Kadowaki K, Yonekubo S, Shibata T, Sato I. 1999. D- and L-Quartz-promoted highly enantioselective synthesis of a chiral compound. J Am Chem Soc 121: 11235-11236.

Strecker A. 1850. Ueber die künstliche Bildung der Milchsäure und einen neuen, dem Glycocoll homologen Körper. Ann Chem Pharm 75: 2745.

van't Hoff JH. 1874. Voorstel tot uitbredning der tegenwoordig. J. Greven, Utrecht.

Viedma C. 2005. Chiral symmetry breaking during crystallization: Complete chiral purity induced by nonlinear autocatalysis and recycling. Phys Rev Lett 94: 065504.

Viedma C, Ortiz JE, de Torres T, Izumi T, Blackmond DG. 2008. Evolution of solid phase homochirality for a proteinogenic amino acid. J Am Chem Soc 130: 15274-15275.

von Kiedrowski G. 2005. Systems Chemistry, Workshop at the European Center for Living Technology, Venice International University, Venice, Oct 3-4, 2005.

Wagner AJ, Zubarev D Yu, Asparu-Guzik A, Blackmond DG. 2016. Chiral sugars drive enantioenrichment in prebiotic amino acid synthesis. ACS Central Sci 3: 322-328. 


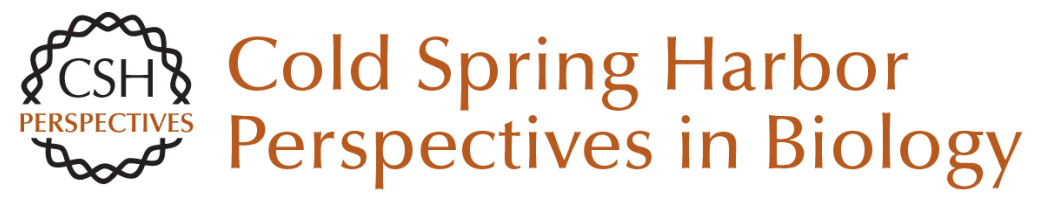

\section{The Origin of Biological Homochirality}

Donna G. Blackmond

Cold Spring Harb Perspect Biol 2019; doi: 10.1101/cshperspect.a032540

\section{Subject Collection RNA Worlds}

Alternate RNA Structures

Marie Teng-Pei Wu and Victoria D'Souza

Approaches for Understanding the Mechanisms

of Long Noncoding RNA Regulation of Gene

Expression

Patrick McDonel and Mitchell Guttman

Principles and Practices of Hybridization Capture

Experiments to Study Long Noncoding RNAs That

Act on Chromatin

Matthew D. Simon and Martin Machyna

Linking RNA Sequence, Structure, and Function

on Massively Parallel High-Throughput

Sequencers

Sarah K. Denny and William J. Greenleaf

Extensions, Extra Factors, and Extreme

Complexity: Ribosomal Structures Provide

Insights into Eukaryotic Translation

Melanie Weisser and Nenad Ban

Nascent RNA and the Coordination of Splicing with Transcription

Karla M. Neugebauer

Combining Mass Spectrometry (MS) and Nuclear Magnetic Resonance (NMR) Spectroscopy for Integrative Structural Biology of Protein-RNA Complexes

Alexander Leitner, Georg Dorn and Frédéric H.-T. Allain
Structural Biology of Telomerase

Yaqiang Wang, Lukas Susac and Juli Feigon

Structural Insights into Nuclear pre-mRNA

Splicing in Higher Eukaryotes

Berthold Kastner, Cindy L. Will, Holger Stark, et al.

What Are 3' UTRs Doing?

Christine Mayr

Single-Molecule Analysis of Reverse

Transcriptase Enzymes

Linnea I. Jansson and Michael D. Stone

CRISPR Tools for Systematic Studies of RNA

Regulation

Jesse Engreitz, Omar Abudayyeh, Jonathan

Gootenberg, et al.

Relating Structure and Dynamics in RNA Biology Kevin P. Larsen, Junhong Choi, Arjun Prabhakar, et al.

Beyond DNA and RNA: The Expanding Toolbox of Synthetic Genetics

Alexander I. Taylor, Gillian Houlihan and Philipp Holliger

For additional articles in this collection, see http://cshperspectives.cshlp.org/cgi/collection/

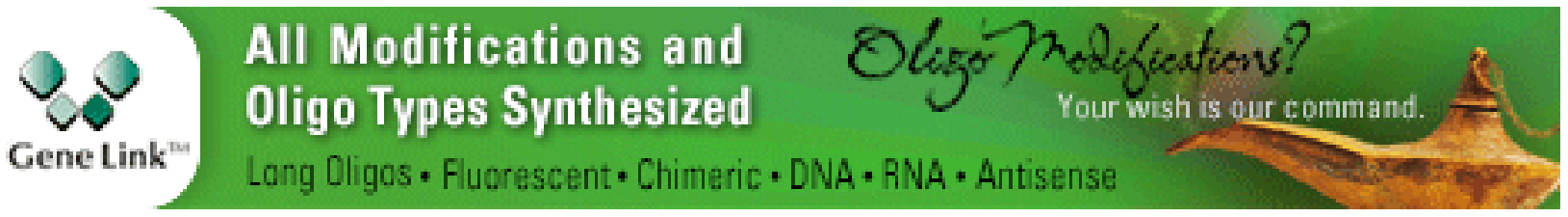

Copyright @ 2019 Cold Spring Harbor Laboratory Press; all rights reserved 
Discovering and Mapping the Modified Nucleotides That Comprise the Epitranscriptome of mRNA

Bastian Linder and Samie R. Jaffrey
Structural Basis of Nuclear pre-mRNA Splicing:

\section{Lessons from Yeast}

Clemens Plaschka, Andrew J. Newman and Kiyoshi Nagai

For additional articles in this collection, see http://cshperspectives.cshlp.org/cgi/collection/

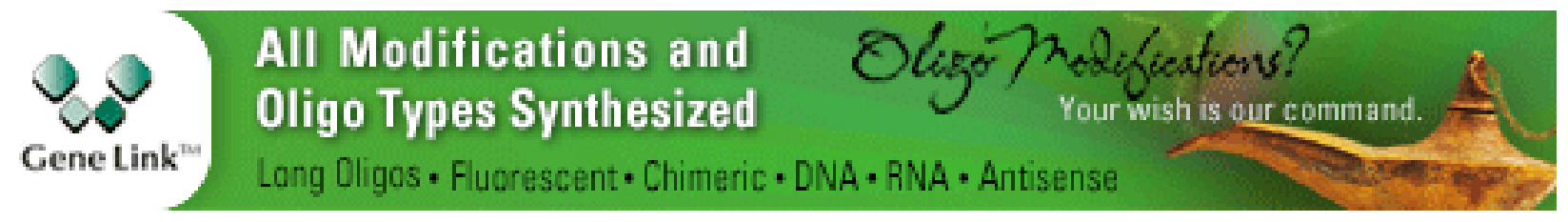

Copyright (C) 2019 Cold Spring Harbor Laboratory Press; all rights reserved 